\title{
Recurrence Relations for Moments of Generalized Order Statistics from Marshall - Olkin extended Kumaraswamy distribution and its Characterization
}

\author{
Ibrahim B. Abdul-Moniem* \\ Department of Quantitative Methods, College of Commerce - Aswan University, Aswan, Egypt \\ *Corresponding author: ibtaib@hotmail.com
}

Received April 28, 2014; Revised May 15, 2014; Accepted September 24, 2014

\begin{abstract}
In this paper, Marshall - Olkin extended Kumaraswamy distribution will be obtained. We give some properties for this distribution. Recurrence relations for single and product moments of generalized order statistics from Marshall - Olkin extended Kumaraswamy distribution have been obtained. Specializations to order statistics and records have been made. Further, using a recurrence relation for single moments we obtain characterization of Marshall - Olkin extended Kumaraswamy distribution.
\end{abstract}

Keywords: Kumaraswamy distribution, marshall, Olkin extended distributions, generalized order statistics, order statistics, records, single and product moments, recurrence relations, characterization

Cite This Article: I.B. Abdul-Moniem, "Recurrence Relations for Moments of Generalized Order Statistics from Marshall - Olkin extended Kumaraswamy distribution and its Characterization.” American Journal of Applied Mathematics and Statistics, vol. 2, no. 5 (2014): 324-329. doi: 10.12691/ajams-2-5-5.

\section{Introduction}

A random variable $X$ is said to have a Kumaraswamy distribution (KD) if its probability density function is ( $p d f)$ in the form:

$$
\begin{aligned}
& f(x ; \lambda, \beta)=\lambda \beta x^{\lambda-1}\left(1-x^{\lambda}\right)^{\beta-1} ; \\
& 0 \leq x \leq 1, \quad \lambda, \beta>0
\end{aligned}
$$

The cumulative distribution func-tion $(C D F)$ and survival function $(S F)$ are:

$$
\begin{aligned}
& F(x ; \lambda, \beta)=1-\left(1-x^{\lambda}\right)^{\beta} ; \\
& 0 \leq x \leq 1, \quad \lambda, \beta>0
\end{aligned}
$$

and

$$
\begin{aligned}
& \bar{F}(x ; \lambda, \beta)=\left(1-x^{\lambda}\right)^{\beta} ; \\
& 0 \leq x \leq 1, \quad \lambda, \beta>0
\end{aligned}
$$

More details on this distribu-tion and its applications can be found in Kumaraswamy [19], Sundar and Subbiah [24], Fletcher and Ponnambalam [10], Seifi et al. [23], Ganji et al. [11], Sanchez et al. [22] and Courard-Hauri [9].

Marshall and Olkin [19] intr-oduced a new method of adding a parameter into a family of distrib-utions. According to them if $\bar{F}(x)$ denote the survival or reliability function of a continuous random variable $X$ then the timely honored device of adding a new parameter results in another survival function $\bar{G}(x)$ defined by

$$
\begin{aligned}
& \bar{G}(x)=\frac{\alpha \bar{F}(x)}{1-\bar{\alpha} \bar{F}(x)} ;-\infty<x<\infty, \alpha>0 \\
& \text { and } \bar{\alpha}=1-\alpha .
\end{aligned}
$$

The $p d f$ and hazard rate function (HRF) corresponding to $\bar{G}(x)$ are:

$$
\begin{aligned}
& g(x)=\frac{\alpha f(x)}{[1-\bar{\alpha} \bar{F}(x)]^{2}} ;-\infty<x<\infty, \\
& \alpha>0 \text { and } \bar{\alpha}=1-\alpha,
\end{aligned}
$$

and

$$
\begin{aligned}
& r(x)=\frac{\alpha h(x)}{1-\bar{\alpha} \bar{F}(x)} ;-\infty<x<\infty, \\
& \alpha>0 \text { and } \bar{\alpha}=1-\alpha,
\end{aligned}
$$

where, $h(x)$ is the HRF correspo-nding $f(x)$.

Here, we substituting from (1.1) and (1.3) in (1.5) to obtain a new distribution called Marshall-Olkin extended Kumaraswamy distribu-tion $(M-O E K D)$ as follows

$$
\begin{aligned}
& g(x)=\frac{\alpha \lambda \beta x^{\lambda-1}\left(1-x^{\lambda}\right)^{\beta-1}}{\left[1-\bar{\alpha}\left(1-x^{\lambda}\right)^{\beta}\right]^{2}} ; 0 \leq x \leq 1, \\
& \lambda, \beta>0 \text { and } \bar{\alpha}=1-\alpha
\end{aligned}
$$

The $C D F, S F$ and $H R F$ corresponding to $g(x)$ are: 
$G(x)=\frac{\alpha\left(1-x^{\lambda}\right)^{\beta}}{1-\bar{\alpha}\left(1-x^{\lambda}\right)^{\beta}} ; 0 \leq x \leq 1$,

$\lambda, \beta>0$ and $\bar{\alpha}=1-\alpha$

$\bar{G}(x)=\frac{\alpha\left(1-x^{\lambda}\right)^{\beta}}{1-\bar{\alpha}\left(1-x^{\lambda}\right)^{\beta}} ; 0 \leq x \leq 1$,

$\lambda, \beta>0$ and $\bar{\alpha}=1-\alpha$

and

$$
r(x)=\frac{\lambda \beta x^{\lambda-1}}{\left(1-x^{\lambda}\right)\left[1-\bar{\alpha}\left(1-x^{\lambda}\right)^{\beta}\right]}
$$

$0 \leq x \leq 1, \lambda, \theta>0$ and $\bar{\alpha}=1-\alpha$

In view of (1.7) and (1.9), we have

$$
\begin{aligned}
& \bar{G}(x)=\frac{g(x)}{\lambda \beta}\left[\left(x^{1-\lambda}-x\right)-\bar{\alpha} \sum_{i=1}^{\infty}\left(\begin{array}{l}
\beta \\
i
\end{array}\right)\right. \\
& \left.(-1)^{i}\left(x^{1-\lambda(1-i)}-x^{1+\lambda i}\right)\right]
\end{aligned}
$$

The inverse of the distribution function (1.8) yields a very simple quantile function

$$
Q(y)=\left[1-\left(\frac{1-y}{1-\bar{\alpha} y}\right)^{\frac{1}{\beta}}\right]^{\frac{1}{\lambda}} ; y \in(0,1)
$$

which facilitates ready quantile based statistical modeling. In addition, $Q(y)$ gives a trivial random variable generation. If $U \sim u(0,1)$, then $X \sim M-\operatorname{OEKD}(\alpha, \beta, \lambda)$ is given by

$$
X=\left[1-\left(\frac{1-U}{1-\bar{\alpha} U}\right)^{\frac{1}{\beta}}\right]^{\frac{1}{\lambda}} .
$$

The mode for $M-O E K D$ can be obtained as the root of the following equation:

$$
\begin{array}{r}
(\lambda-1)-x^{\lambda}(\lambda \beta-1)-\bar{\alpha}\left(1-x^{\lambda}\right)^{\beta} \\
{\left[x^{\lambda}(\lambda \beta+1)+(\lambda-1)\right]=0}
\end{array}
$$

and the median for $M-O E K D$ is

$$
X=\left[1-\left(\frac{1}{\alpha+1}\right)^{\frac{1}{\beta}}\right]^{\frac{1}{\lambda}}
$$

This distribution can be applied on some real percentage data. Carrasco et al [16] applied Generalized Kumaraswamy Distribution on the observed percentage of children living in households with per capita income less than R\$ 75.50 in 1991 in 5509 Brazilian municipal districts.
The concept of generalized order statistics (gos) was introduced by Kamps [13]. A variety of order models of random variables is contained in this concept.

Let, for simplicity, $F$ throughout denote an absolutely continuous distribution function with density function $f$. The random variables $X(1, n, \tilde{m}, k), \ldots, X(n, n, \tilde{m}, k)$ are called generalized order statistics based on $F$, if their joint $p d f$ of the form

$$
k\left(\prod_{j=1}^{n-1} \gamma_{j}\right)\left(\prod_{i=1}^{n-1}\left[\bar{F}\left(x_{i}\right)\right]^{m_{i}} f\left(x_{i}\right)\right)
$$

for

$$
\left[\bar{F}\left(x_{n}\right)\right]^{k-1} f\left(x_{n}\right)
$$

$F^{-1}(1)>x_{1} \geq x_{2} \geq \ldots \geq x_{n}>F^{-1}(0)$, with parameters $n \in N, n \geq 2, k>0, \quad \tilde{m}=\left(m_{1}, m_{2}, \ldots, m_{n-1}\right) \in R^{n-1}$, $M_{r}=\sum_{i=r}^{n-1} m_{i}$, such that $\gamma_{r}=k+n-r+M_{r}>0$, for all $r \in\{1,2, \ldots, n-1\}$.

For $\gamma_{i} \neq \gamma_{j}, i \neq j$ for all $i, j \in(1,2, \ldots, n)$ the $p d f$ of $X(r, n, \tilde{m}, k)$ is given by Kamps and Cramer [14] in the following way

$$
f_{X(r, n, \tilde{m}, k)}(x)=C_{r-1} f(x) \sum_{i=1}^{r} a_{i}(r)[\bar{F}(x)]^{\gamma_{i}-1}(1.16)
$$

The joint $p d f$ of $X(r, n, \tilde{m}, k)$ and $X(s, n, \tilde{m}, k)$, $1 \leq r<s \leq n$ is given as

$$
\begin{aligned}
& f_{X(r, n, \tilde{m}, k), X(s, n, \tilde{m}, k)}(x, y)= \\
& C_{s-1}\left(\sum_{i=r+1}^{s} a_{i}^{(r)}(s)\left[\frac{\bar{F}(y)}{\bar{F}(x)}\right]^{\gamma_{i}}\right) \\
& \left(\sum_{i=1}^{r} a_{i}(r)[\bar{F}(x)]^{\gamma_{i}}\right) \frac{f(x)}{\bar{F}(x)} \frac{f(y)}{\bar{F}(y)},
\end{aligned}
$$

where $x<y$ and

$$
\begin{aligned}
& a_{i}(r)=\prod_{\substack{j=1 \\
j \neq i}}^{r} \frac{1}{\gamma_{j}-\gamma_{i}}, \quad 1 \leq i \leq r \leq n, \\
& a_{i}^{(r)}(s)=\prod_{\substack{j=r+1 \\
j \neq i}}^{s} \frac{1}{\gamma_{j}-\gamma_{i}}, \quad r+1 \leq i \leq s \leq n .
\end{aligned}
$$

It may be noted that for $m_{1}=m_{2}=\ldots=m_{n-1}=m \neq-1$,

$$
a_{i}(r)=\frac{(-1)^{r-i}}{(m+1)^{r-1}(r-1) !}\left(\begin{array}{c}
r-1 \\
r-i
\end{array}\right)
$$

and

$$
a_{i}^{(r)}(s)=\frac{(-1)^{s-i}}{(m+1)^{s-r-1}(s-r-1) !}\left(\begin{array}{c}
s-r-1 \\
s-i
\end{array}\right) .
$$

Therefore $p d f$ of $X(r, n, \tilde{m}, k)$ given in (16) reduces to $f_{X(r, n, m, k)}(x)=\frac{C_{r-1}}{(r-1) !}[\bar{F}(x)]^{\gamma_{r}-1} f(x) g_{m}^{r-1}[F(x)],(1.20)$ 
and joint $p d f$ of $X(r, n, \tilde{m}, k)$ and $X(s, n, \tilde{m}, k)$ given in (1.17) reduces to

$$
\begin{aligned}
& f_{X(r, n, m, k), X(s, n, m, k)}(x, y)=\frac{C_{s-1}}{(r-1) !(s-r-1) !} \\
& {[\bar{F}(x)]^{m} f(x) g_{m}^{r-1}[F(x)]} \\
& \left\{h_{m}[F(y)]-h_{m}[F(x)]\right\}^{s-r-1} \\
& {[\bar{F}(y)]^{\gamma_{s}-1} f(y), \quad x<y}
\end{aligned}
$$

where

$$
\begin{aligned}
& C_{r-1}=\prod_{i=1}^{r} \gamma_{i}, \gamma_{i}=k+(n-i)(m+1) \\
& h_{m}(x)= \begin{cases}\frac{-1}{m+1} x^{m+1}, & m \neq-1 \\
-\ln x, & m=-1\end{cases}
\end{aligned}
$$

and

$$
g_{m}(x)=h_{m}(x)-h_{m}(1), \quad x \in[0,1) .
$$

We shall also take $X(0, n, m, k)=0$. If $m=0, k=1$, then $X(r, n, m, k)$ reduces to the $(n-r+1)^{t h}$ order statistics, $X_{n-r+1: n}$ from the sample $X_{1}, X_{2}, \ldots, X_{n}$ and when $\mathrm{m}=-1$, then $X(r, n, m, k)$ reduces to the $k^{\text {th }}$ record values (Pawlas and Szynal [21]).

Many authors utilized the gos in their work, such as Kamps and Gather [15], Athar et al [7], Cramer and Kamps [8], Ahsanullah [4], Pawlas and Szynal [21], Ahmed [2], Ahmed and Fawzy [3], Khan et al. [17], ALHussaini et al. [5] and Kumar [18]. Abdul-Moniem [1] obtained recurrence relations for moments of lower gos from exponentiated Lomax distribution and its characterization.

In this paper, we have established explicit expressions and some recurrence relations for single and product moments of gos from MOEKD family of life distributions. Further its various deductions and particular cases are discussed. Characterization of M-OEKD has been obtained on using a recurrence relation for single moments.

\section{Single Moments of gos}

Theorem 2.1. let $X$ be a random variable has $p d f(7)$. Then for integer $j$ such that $j>0$, the follow-ing recurrence relation is satisfied.

$$
\begin{gathered}
E\left[X^{j}(r, n, \tilde{m}, k)\right]=\frac{1}{\lambda \beta \gamma_{r}+j} \\
\left\{\lambda \beta \gamma_{r} E\left[X^{j}(r-1, n, \tilde{m}, k)\right]+\right. \\
\left.j E\left[X^{j-\lambda}(r, n, \tilde{m}, k)\right]\right\}-\frac{j \bar{\alpha}}{\lambda \beta \gamma_{r}+j} \\
\sum_{i=0}^{\infty}\left(\begin{array}{l}
\beta \\
i
\end{array}\right)(-1)^{i}\left\{E\left[X^{j-\lambda(1-i)}(r, n, \tilde{m}, k)\right]\right. \\
\left.+E\left[X^{j+\lambda i}(r, n, \tilde{m}, k)\right]\right\}
\end{gathered}
$$

Proof. We have from Lemma 2.3 (Athar and Islam [6]) that

$$
\begin{aligned}
& E[\xi\{X(r, n, \tilde{m}, k)\}]- \\
& E[\xi\{X(r-1, n, \tilde{m}, k)\}] \\
& =C_{r-2} \int_{\theta}^{\beta} \xi^{\prime}(x) \sum_{i=1}^{r} a_{i}(r)[\bar{F}(x)]^{\gamma_{i}} d x
\end{aligned}
$$

If we let $\xi(x)=x^{j}$, then

$$
\begin{aligned}
& E\left[X^{j}(r, n, \tilde{m}, k)\right]-E\left[X^{j}(r-1, n, \tilde{m}, k)\right] \\
& =j C_{r-2} \int_{\theta}^{\beta} X^{j-1} \sum_{i=1}^{r} a_{i}(r)[\bar{F}(x)]^{\gamma_{i}} d x
\end{aligned}
$$

On using (1.11) in (2.2), we get

$$
\begin{aligned}
& E\left[X^{j}(r, n, \tilde{m}, k)\right]- \\
& E\left[X^{j}(r-1, n, \tilde{m}, k)\right]=\frac{j}{\lambda \beta \gamma_{r}} C_{r-1} \\
& \int_{0}^{1} X^{j-1} \sum_{i=1}^{r} a_{i}(r)[\bar{F}(x)]^{\gamma_{i}-1}\left[\left(x^{1-\lambda}-x\right)-\right. \\
& \left.\bar{\alpha} \sum_{i=1}^{\infty}\left(\begin{array}{l}
\beta \\
i
\end{array}\right)(-1)^{i}\left(x^{1-\lambda(1-i)}-x^{1+\lambda i}\right)\right] f(x) d x \\
& =\frac{j}{\lambda \beta \gamma_{r}} C_{r-1} \int_{0}^{1}\left(x^{j-\lambda}-x^{j}\right) \sum_{i=1}^{r} a_{i}(r) \\
& {[\bar{F}(x)]^{\gamma_{i}-1} f(x) d x-} \\
& \frac{j \bar{\alpha}}{\lambda \beta \gamma_{r}} C_{r-1} \sum_{i=1}^{\infty}(\beta)(-1)^{i} \int_{0}^{1}\left(x^{j-\lambda(1-i)}-x^{j+\lambda i}\right) \\
& \sum_{i=1}^{r} a_{i}(r)[\bar{F}(x)]^{\gamma_{i}-1} f(x) d x
\end{aligned}
$$

Which after simplification leads to (1.2).

Corollary 2.2. For $m_{1}=m_{2}=\ldots=m_{n-1}=m \neq-1$, the recurrence relations for single moment of gos for $M$ $\mathrm{OEKD}$ is given as

$$
\begin{gathered}
E\left[X^{j}(r, n, m, k)\right]=\frac{1}{\lambda \beta \gamma_{r}+j} \\
\left\{\lambda \beta \gamma_{r} E\left[X^{j}(r-1, n, m, k)\right]+\right. \\
\left.j E\left[X^{j-\lambda}(r, n, m, k)\right]\right\}-\frac{j \bar{\alpha}}{\lambda \beta \gamma_{r}+j} \\
\sum_{i=0}^{\infty}\left(\begin{array}{l}
\beta \\
i
\end{array}\right)(-1)^{i}\left\{E\left[X^{j-\lambda(1-i)}(r, n, m, k)\right]\right. \\
\left.+E\left[X^{j+\lambda i}(r, n, m, k)\right]\right\}
\end{gathered}
$$

Proof. This can easy be deduced from (2.1) in view of the relation (1.18).

Note that: We can obtain the recurrence relations for single moment of gos for Kumaraswamy distribution by taking $\alpha=1$ in (2.3), established by Kumar [18].

Remark 2.3 Putting $m=0, k=1$ in (2.3), we obtain recurrence relations for single moments of order statistics as 


$$
\begin{aligned}
& E\left[X_{r: n}^{j}\right]=\frac{\lambda \beta(n-r+1)}{\lambda \beta(n-r+1)+j} \\
& E\left[X_{r-1: n}^{j}\right]+\frac{j}{\lambda \beta(n-r+1)+j} \\
& \left\{E\left[X_{r: n}^{j-\lambda}\right]-\bar{\alpha} \sum_{l=0}^{\infty}\left(\begin{array}{l}
\beta \\
l
\end{array}\right)(-1)^{l}\right. \\
& \left.\left[E\left[X_{r: n}^{j-\lambda(1-l)}\right]-E\left[X_{r: n}^{j+\lambda l}\right]\right]\right\}
\end{aligned}
$$

Remark 2.4 Setting $m=-1, k \geq 1$ in (2.3), we obtain the recurrence relations of upper $K$-record values as

$$
\begin{aligned}
& E\left[\left(X_{U(n)}^{j}\right)^{k}\right]=\frac{\lambda \beta k}{\lambda \beta k+j} E\left[\left(X_{U(n-1)}^{j}\right)^{k}\right] \\
& +\frac{j}{\lambda \beta k+j}\left\{E\left[\left(X_{U(n)}^{j-\lambda}\right)^{k}\right]-\bar{\alpha} \sum_{l=0}^{\infty}\left(\begin{array}{l}
\beta \\
l
\end{array}\right)(-1)^{l}\right. \\
& \left.\left[E\left[\left(X_{U(n)}^{j-\lambda(1-l)}\right)^{k}\right]-E\left[\left(X_{U(n)}^{j+\lambda l}\right)^{k}\right]\right]\right\}
\end{aligned}
$$

\section{Product Moments of gos}

Theorem 3.1 let $X$ be a random variable has $p d f$ (1.7). Then for integer $i, j$ such th-at $i, j>0$, the following recurrence relation is satisfied.

$$
\begin{aligned}
& E\left[X^{i}(r, n, \tilde{m}, k) \cdot X^{j}(s, n, \tilde{m}, k)\right]= \\
& \frac{\gamma_{s} \lambda \beta}{\gamma_{s} \lambda \beta+j} E\left[X^{i}(r, n, \tilde{m}, k) X^{j}(s-1, n, \tilde{m}, k)\right]+\frac{j}{\gamma_{s} \lambda \beta+j} \\
& \left\{E\left[X^{i}(r, n, \tilde{m}, k) X^{j-\lambda}(s, n, \tilde{m}, k)\right]\right. \\
& -\bar{\alpha} \sum_{l=0}^{\infty} \frac{(-1)^{l}}{l !} E\left[\begin{array}{l}
X^{i}(r, n, \tilde{m}, k) \\
X^{j-\lambda(1-l)}(s, n, \tilde{m}, k)
\end{array}\right] \\
& \left.-E\left[X^{i}(r, n, \tilde{m}, k) \cdot X^{j+\lambda l}(s, n, \tilde{m}, k)\right]\right\}
\end{aligned}
$$

Proof. We have from Lemma 3.2 (Athar and Islam [6]) that

$$
\begin{gathered}
E[\xi\{X(r, n, \tilde{m}, k) \cdot X(s, n, \tilde{m}, k)\}]- \\
E[\xi\{X(r, n, \tilde{m}, k) \cdot X(s-1, n, \tilde{m}, k)\}]= \\
C_{s-2} \int_{\theta}^{\beta} \int_{x}^{\beta} \frac{\partial}{\partial y} \xi(x, y) \sum_{l=r+1}^{s} a_{l}^{(r)}(s)\left[\frac{\bar{F}(y)}{\bar{F}(x)}\right]^{\gamma_{l}} \\
\sum_{l=1}^{r} a_{l}(r)[\bar{F}(x)]^{\gamma_{l}} \frac{f(x)}{\bar{F}(x)} d y d x
\end{gathered}
$$

If we let $\xi(x, y)=x^{i} y^{j}$, then

$$
\begin{gathered}
E\left[X^{i}(r, n, \tilde{m}, k) \cdot X^{j}(s, n, \tilde{m}, k)\right]- \\
E\left[X^{i}(r, n, \tilde{m}, k) \cdot X^{j}(s-1, n, \tilde{m}, k)\right]= \\
\frac{j C_{s-1}}{\gamma_{s}} \int_{0}^{\infty} \int_{x}^{\infty} x^{i} y^{j-1} \sum_{l=r+1}^{s} a_{l}^{(r)}(s)\left[\frac{\bar{F}(y)}{\bar{F}(x)}\right]^{\gamma_{l}} \\
\sum_{l=1}^{r} a_{l}(r)[\bar{F}(x)]^{\gamma_{l}} \frac{f(x)}{\bar{F}(x)} d y d x
\end{gathered}
$$

In view of (1.11), note that

$$
\begin{aligned}
& \frac{\bar{F}(y)}{f(y)}=\frac{1}{\lambda \beta}\left[\left(y^{1-\lambda}-y\right)-\right. \\
& \left.\bar{\alpha} \sum_{l=1}^{\infty}\left(\begin{array}{l}
\beta \\
l
\end{array}\right)(-1)^{l}\left(y^{1-\lambda(1-l)}-y^{1+\lambda l}\right)\right]
\end{aligned}
$$

Therefore,

$$
\begin{aligned}
& E\left[X^{i}(r, n, \tilde{m}, k) \cdot X^{j}(s, n, \tilde{m}, k)\right]- \\
& E\left[X^{i}(r, n, \tilde{m}, k) \cdot X^{j}(s-1, n, \tilde{m}, k)\right]= \\
& \frac{j C_{s-1}}{\gamma_{s} \lambda \beta}\left\{\int_{0}^{1} \int_{x}^{1} x^{i} y^{j-\lambda} \sum_{l=r+1}^{s} a_{l}^{(r)}(s)\left[\frac{\bar{F}(y)}{\bar{F}(x)}\right]^{\gamma_{l}}\right. \\
& \sum_{l=1}^{r} a_{l}(r)[\bar{F}(x)]^{\gamma_{l}} \frac{f(x)}{\bar{F}(x)} \frac{f(y)}{\bar{F}(y)} d y d x \\
& -\int_{0}^{1} \int_{x}^{1} x^{i} y^{j} \sum_{l=r+1}^{s} a_{l}^{(r)}(s)\left[\frac{\bar{F}(y)}{\bar{F}(x)}\right]^{\gamma_{l}} \\
& \left.\sum_{l=1}^{r} a_{l}(r)[\bar{F}(x)]^{\gamma_{l}} \frac{f(x)}{\bar{F}(x)} \frac{f(y)}{\bar{F}(y)} d y d x\right\} \\
& -\frac{j C_{s-1} \bar{\alpha}}{\gamma_{s} \lambda \beta} \sum_{l=1}^{\infty}\left(\begin{array}{c}
\beta \\
l
\end{array}\right)(-1)^{l}\left\{\int_{0}^{1} \int_{x}^{1} x^{i} y^{j-\lambda(1-l)}\right. \\
& \sum_{l=r+1}^{s} a_{l}^{(r)}(s)\left[\frac{\bar{F}(y)}{\bar{F}(x)}\right]^{r i} \sum_{l=1}^{r} a_{l}(r)[\bar{F}(x)]^{r i} \\
& \frac{f(x)}{\bar{F}(x)} \frac{f(y)}{\bar{F}(y)} d y d x-\int_{0}^{1} \int_{x}^{1} x^{i} y^{j-\lambda l} \\
& \sum_{l=r+1}^{s} a_{l}^{(r)}(s)\left[\frac{\bar{F}(y)}{\bar{F}(x)}\right]^{r i} \\
& \left.\sum_{l=1}^{r} a_{l}(r)[\bar{F}(x)]^{\gamma_{l}} \frac{f(x)}{\bar{F}(x)} \frac{f(y)}{\bar{F}(y)} d y d x\right\}
\end{aligned}
$$

Which after simplification leads to (3.1).

Corollary 3.2. For $m_{1}=m_{2}=\ldots=m_{n-1}=m \neq-1$, the recurrence relations for product moments of gos for $\mathrm{M}$ $\mathrm{OEKD}$ is given as

$$
\begin{aligned}
& E\left[X^{i}(r, n, m, k) \cdot X^{j}(s, n, m, k)\right]= \\
& \frac{\gamma_{s} \lambda \beta}{\gamma_{s} \lambda \beta+j} E\left[X^{i}(r, n, m, k) \cdot X^{j}(s-1, n, m, k)\right] \\
& +\frac{j}{\gamma_{s} \lambda \beta+j}\left\{E \left[X^{i}(r, n, m, k)\right.\right. \\
& X^{j-\lambda}(s, n, m, k)-\bar{\alpha} \sum_{l=0}^{\infty} \frac{(-1)^{l}}{l !} \\
& E\left[X^{i-\lambda}(r, n, m, k) X^{j-\lambda(1-l)}(s, n, m, k)\right] \\
& \left.-E\left[X^{i}(r, n, m, k) \cdot X^{j+\lambda l}(s, n, m, k)\right]\right\}
\end{aligned}
$$

Proof. This can easy be deduced from (3.1) in view of the relation (1.19). 
Note that: We can obtain the recurrence relations for product moments of gos for Kumaraswamy distribution by taking $\alpha=1$ in (3.2), established by Kumar [18].

Remark 3.3 Putting $m=0, k=1$ in (3.2), we obtain recurrence relations for product moments of order statistics as

$$
\begin{aligned}
& E\left[X_{r, s: n}^{i, j}\right]=\frac{\lambda \beta(n-s-1)}{\lambda \beta(n-s-1)+j} E\left[X_{r, s-1: n}^{i, j}\right] \\
& +\frac{j}{\lambda \beta(n-s-1)+j}\left\{E\left[X_{r, s: n}^{i, j-\lambda}\right]-\bar{\alpha} \sum_{l=0}^{\infty} \frac{(-1)^{l}}{l !}\right. \\
& \left.\left[E\left[X_{r, s: n}^{i, j-\lambda(1-l)}\right]-E\left[X_{r, s: n}^{i, j+\lambda l}\right]\right]\right\}
\end{aligned}
$$

Remark 3.4 Setting $m=-1$ in (3.2), we obtain the recurrence relations for product moments of $k^{\text {th }}$ record values as

$$
\begin{aligned}
& E\left[\left(X_{r}^{(k)}\right)^{i}\left(X_{s}^{(k)}\right)^{j}\right]=\frac{\lambda \beta k}{\lambda \beta k+j} \\
& E\left[\left(X_{r}^{(k)}\right)^{i}\left(X_{s-1}^{(k)}\right)^{j}\right]+\frac{j}{\lambda \beta k+j} \\
& \left\{E\left[\left(X_{r}^{(k)}\right)^{i}\left(X_{s}^{(k)}\right)^{j-\lambda}\right]-\bar{\alpha} \sum_{l=0}^{\infty} \frac{(-1)^{l}}{l !}\right. \\
& {\left[E\left[\left(X_{r}^{(k)}\right)^{i}\left(X_{s}^{(k)}\right)^{j-\lambda(1-l)}\right]-\right.} \\
& \left.\left.E\left[\left(X_{r}^{(k)}\right)^{i}\left(X_{s}^{(k)}\right)^{j+\lambda l}\right]\right]\right\}
\end{aligned}
$$

\section{Characterization}

Theorem 4.1 Let $X$ be a non-negative random variable having an absolutely continuous distribution function $F(x)$ with $F(0)=0$ and $0<F(x)<1$ for all $x>0$, then

$$
\begin{gathered}
E\left[X^{j}(r, n, m, k)\right]=\frac{1}{\lambda \beta \gamma_{r}+j} \\
\left\{\lambda \beta \gamma_{r} E\left[X^{j}(r-1, n, m, k)\right]+\right. \\
\left.j E\left[X^{j-\lambda}(r, n, m, k)\right]\right\}-\frac{j \bar{\alpha}}{\lambda \beta \gamma_{r}+j} \\
\sum_{i=0}^{\infty}\left(\begin{array}{l}
\beta \\
i
\end{array}\right)(-1)^{i}\left\{E\left[X^{j-\lambda(1-i)}(r, n, m, k)\right]\right. \\
\left.+E\left[X^{j+\lambda i}(r, n, m, k)\right]\right\}
\end{gathered}
$$$$
\text { if and only if } \bar{F}(x)=\frac{\alpha\left(1-x^{\lambda}\right)^{\beta}}{1-\bar{\alpha}\left(1-x^{\lambda}\right)^{\beta}} \text {. }
$$

Proof The necessary part follows immediately from equation (2.3). On the other hand if the recurrence relation in equation (4.1) is satisfied, then on using equation (1.20), we have

$$
\begin{aligned}
& \frac{C_{r-1}}{(r-1) !} \int_{0}^{1} x^{j}[\bar{F}(x)]^{\gamma_{r}-1} f(x) g_{m}^{r-1}[F(x)] d x \\
& =\frac{1}{\lambda \beta \gamma_{r}+j}\left\{\frac{\lambda \beta \gamma_{r} C_{r-2}}{(r-2) !} \int_{0}^{1} x^{j}[\bar{F}(x)]^{\gamma_{r}+m}\right. \\
& f(x) g_{m}^{r-2}[F(x)] d x+\frac{j C_{r-1}}{(r-1) !} \\
& \left.\int_{0}^{1} x^{j-\lambda}[\bar{F}(x)]^{\gamma_{r}-1} f(x) g_{m}^{r-1}[F(x)] d x\right\} \\
& -\frac{j \bar{\alpha} C_{r-1}}{\left(\lambda \beta \gamma_{r}+j\right)(r-1) !} \sum_{i=0}^{\infty}\left(\begin{array}{l}
\beta \\
i
\end{array}\right)(-1)^{i} \\
& \left\{\begin{array}{l}
1 \\
\int_{0}^{1} x^{j-\lambda(1-i)}[\bar{F}(x)]^{\gamma_{r}-1} f(x) g_{m}^{r-1}[F(x)] d x \\
\left.-\int_{0}^{1} x^{j+\lambda l}[\bar{F}(x)]^{\gamma_{r}-1} f(x) g_{m}^{r-1}[F(x)] d x\right\}
\end{array}\right.
\end{aligned}
$$

Integrating the first integral on the right hand side of the above equation, by parts, we get

$$
\begin{aligned}
& \frac{C_{r-1}}{(r-1) !} \int_{0}^{1} x^{j}[\bar{F}(x)]^{\gamma_{r}-1} f(x) g_{m}^{r-1}[F(x)] d x \\
& =\frac{C_{r-1}}{\left(\lambda \beta \gamma_{r}+j\right)(r-1) !} \\
& \left\{\lambda \beta \gamma_{r} \int_{0}^{1} x^{j}[\bar{F}(x)]^{\gamma_{r}-1} f(x) g_{m}^{r-1}[F(x)] d x\right. \\
& -j \lambda \beta \int_{0}^{1} x^{j-1}[\bar{F}(x)]^{\gamma_{r}} g_{m}^{r-1}[F(x)] d x \\
& \left.+j \int_{0}^{1} x^{j-\lambda}[\bar{F}(x)]^{\gamma_{r}-1} f(x) g_{m}^{r-1}[F(x)] d x\right\} \\
& -\frac{j \bar{\alpha}}{\lambda \beta \gamma_{r}+j} \sum_{i=0}^{\infty}\left(\begin{array}{l}
\beta \\
i
\end{array}\right)(-1)^{i}\left\{\frac{C_{r-1}}{(r-1) !} \int_{0}^{1} x^{j-\lambda(1-i)}\right. \\
& {[\bar{F}(x)]^{\gamma_{r}-1} f(x) g_{m}^{r-1}[F(x)] d x-\frac{C_{r-1}}{(r-1) !}} \\
& \left.\int_{0}^{1} x^{j+\lambda l}[\bar{F}(x)]^{\gamma_{r}-1} f(x) g_{m}^{r-1}[F(x)] d x\right\}
\end{aligned}
$$

Which is implies that

$$
\begin{gathered}
\frac{j C_{r-1}}{\left(\lambda \beta \gamma_{r}+j\right)(r-1) !} \int_{0}^{1} x^{j-1}[\bar{F}(x)]^{\gamma_{r}-1} \\
g_{m}^{r-1}[F(x)]\{x f(x)+\lambda \beta \bar{F}(x)- \\
x^{1-\lambda} f(x)+\bar{\alpha} \sum_{i=0}^{\infty}\left(\begin{array}{l}
\beta \\
i
\end{array}\right)(-1)^{i} f(x) \\
\left.\left[x^{1-\lambda(1-i)}-x^{1+\lambda i}\right]\right\} d x=0
\end{gathered}
$$

Now applying a generalization of the Muntz-Szasz theorem (Hwang and Lin [12]) to equation (4.2), we get 


$$
\begin{array}{r}
\frac{\bar{F}(x)}{f(x)}=\left[\left(x^{1-\lambda}-x\right)-\bar{\alpha} \sum_{i=0}^{\infty}\left(\begin{array}{l}
\beta \\
i
\end{array}\right)(-1)^{i}\right. \\
\left.\left(x^{1-\lambda(1-i)}-x^{1+\lambda i}\right)\right]
\end{array}
$$

which prove that

$$
\bar{F}(x)=\frac{\alpha\left(1-x^{\lambda}\right)^{\beta}}{1-\bar{\alpha}\left(1-x^{\lambda}\right)^{\beta}}
$$

\section{Conclusion}

Here we propose a new model, the so-called the Marshall - Olkin exte-nded Kumaraswamy distribution which extends the Kumaraswamy distribution. Some properties for this distribution have been obtained. We give recurrence relations for single and product moments of generalized order statistics from Marshall - Olkin extended Kumaraswamy distribution. Specializations to order statistics and records have been made. We obtain characterization of Marshall - Olkin extended Kumaraswamy distribution using a recurrence relation for single mom-ents.

\section{Acknowledgment}

The author is thankful to the Editor and the Referee for their fruitful suggestions and comments.

\section{References}

[1] I.B. Abdul-Moniem, Recurrence re-lations for moments of lower generalized order statistics for-m exponentiated Lomax distribution and its characterization, J. Math. Comput. Sci. 2 (4) (2012), 999-1011.

[2] A.A. Ahmad, Recurrence relations for single and product moments of generalized order statistics from doubly truncated Burr type XII distribution, J. Egypt. Math. Soc. 15 (1) (2007), 117128.

[3] A.A. Ahmad and A.M. Fawzy, Recurrence relations for single moments of generalized order statistics from doubly truncated distribution, J. Statist. Plann. Infer. 117 (2) (2003), 241-249.

[4] M. Ahsanullah, Generalized order statistics from exponential distribution, J. Statist. Plann. Infer. 85 (1-2) (2000), 85-91.

[5] E.K. AL-Hussaini, A.A. Ahmad and M.A. Al-Kashif, Recurrence relations for moment and conditional moment generating functions of generalized order statistics, Metrika 61 (2) (2005), 199-220.
[6] H. Athar and H. Islam, Recurrence relations for single and product moments of generalized order statistics from a general class of distribution, Metron, LXII (3) (2004), 327-337.

[7] Athar, H., Nayabuddin and Khwaja, S. K. (2012) Relations for moments of generalized order statistics from Marshal-Olkin extended Weibull Distribution and its characterization, ProbStat Forum, 5 (4), 127-132.

[8] E. Cramer and U. Kamps, Relations for expectations of functions of generalized order statistics, J. Statist. Plann. Infer. 89 (1-2) (2000), 79-89.

[9] D. Courard-Hauri, Using Monte Carlo analysis to investigate the relation-ship between overconsumption and uncertain access to one's personal utility function. Ecological Economics, 64 (2007), 152-162.

[10] S. C. Fletcher and K. Ponnambalam, Estimation of reservoir yield and storage distribution using moments analysis, Journal of Hydro-logy, 182 (1996), 259-275.

[11] A. Ganji, K. Ponnambalam, D. Khalili, and M. Karamouz, Grain yield reliability analysis with crop water demand uncertainty, Stochastic Environmental Research and Risk Assessment, 20(2006), 259-277.

[12] J.S. Hwang and G.D. Lin, On a Generalized Moment Problem II, Proc. of Amer Math. Soc. 91 (1984), 577-580.

[13] U. Kamps, A Concept of Generalized Order Statistics, B. G. Teubner, Stuttgart, (1995).

[14] U. Kamps and S. Cramer, On distributions of generalized order statistics. Statistics, 35 (2001), 269-280.

[15] U. Kamps and U. Gather, Characteristic property of generalized order statistics for exponential distribution. Appl. Math. (Warsaw), 24 (1997), 383-391.

[16] J. M. F. Carrasco, S. L. P. Ferrari, and G. M. Cordeiro, A New Generalized Kumaraswamy Di-stribution, arXiv: 1004.0911v1 [stat.ME] 6 Apr. (2010).

[17] R. U. Khan, Z. Anwar, and H. Athar, Recurrence relations for single and product moments of generalized order statistics fro-m doubly truncated Weibull distribution, Aligarh J. Statist. 27 (2007), 69-79.

[18] D. Kumar, Generalized order statistics from Kumaraswamy distribution and its characterization. Tamsui Oxford J. Infor. and Math. Sci. 27 (4) (2011), 463-476.

[19] P. Kumaraswamy, Generalized probability density function for double- bounded random-processes, Journal of Hydrology , 46 (1980), 79-88.

[20] A.W. Marshall and I. Olkin, I., A new method for adding a parameter to a family of distributions with application to the exponential and Weibull families, Biometrika 84 (3) (1997), 641652.

[21] P. Pawlas and D. Szynal, Recurrence relations for single and product moments of generalized order statistics from Pareto, generalized Pareto and Burr dist-ributions, Commun. Stat. Theory Methods, 30 (4) (2001), 739-746.

[22] S. Sanchez, J. Ancheyta, and W. C. McCaffrey, Comparison of probability distribution function for fitting distillation curves of petroleum, Energy and Fuels, 21 (2007), 2955-2963.

[23] A. Seifi, K. Ponnambalam, and J. Vlach, Maximization of manufacturing yield of systems with arbitrary distributions of comp-onent values, Annals of Operations Research, 99 (2000), 373-383.

[24] V. Sundar and K. Subbiah, Application of double bounded probability density function for analysis of ocean waves, Ocean Engineering, 16 (1989), 193-200. 